\title{
THE EFFERENT FIBRES OF THE HIPPOCAMPUS IN THE MONKEY
} BY

\author{
D. A. SIMPSON
}

\author{
From the Department of Human Anatomy, University of Oxford
}

Speculations upon the functions of the hippocampus have been numerous in the last few years and have led to some important experimental studies. In the critical review by Brodal (1947) the obscurity surrounding the afferent connexions of the hippocampal complex was stressed. Since the publication of this paper, the hippocampal afferents from the cingulate cortex have been examined experimentally in the rabbit (Adey, 1951) and in the monkey (Adey and Meyer, 1952) by Glees' silver method, and Pribram, Lennox, and Dunsmore (1950) have presented electro-neuronographic experiments on the connexions of the entorhinal and prepiriform areas in the macaque.

The efferent projection of the hippocampus has been the subject of some recent experimental studies, notably those of Sprague and Meyer (1950) on the rabbit, employing the Glees method, and of Allen $(1944,1948)$ upon the dog, using both the Marchi technique and the examination of early retrograde changes. These experimental investigations were made on lower mammals, and earlier workers (von Gudden, 1881; Probst, 1901 ; Edinger and Wallenberg, 1902 ; Yamagata, 1927 ; Le Gros Clark, 1932 ; Gerebtzoff, 1941-42 ; Fox, 1943 ; Allen, 1944) have also used rodents and carnivores (vide infra). The Dejerines (1897), indeed, briefly describe the fornix fibre degeneration in a human brain in which the hippocampus, the splenium, and other structures had been destroyed by cerebral softening. Their technical methods, however, are not adequately described ; moreover, their lesion appears to have been so massive as to make some of their conclusions unreliable. Since the allocortical structures are by no means constant phylogenetically, it seems important to confirm in the primate brain the observations made on subprimates, and to employ methods giving more precise information than is obtainable by the Marchi method used by most of the authors quoted. The experiments reported here were designed to provide accurate knowledge of the termination of the efferent fibres of the hippocampus in the monkey. The findings of previous workers have also been reviewed in some detail, for they appear to demonstrate significant phylogenetic variations in the destiny of these fibres.

\section{Material}

Numerical Examination of Fornix Fibres.-As a preliminary to a detailed investigation of the destiny of the fornix fibres, an attempt was made to estimate what proportion of these fibres reach the mamillary body. An approximate indication of this may be obtained by counting and comparing the number of fibres in the fornix before and after the departure of its precommissural component.

Selected portions of the fornix of a young, healthy macaque were embedded in paraffin wax, cut transversely at $5 \mu$, and stained with Bodian's protargol. Two levels were examined quantitatively : the fornix beneath the corpus callosum, just behind the caudal extremity of the septum lucidum, and the descending column of the fornix in the hypothalamus (Fig. 1a, 1b). In the first, the precommissural fibres are included, while at the latter level, these have presumably left the main bundle. At each level fibres in 10 areas chosen at random were counted, using the camera lucida method recommended by Allison and Chacko (1949), and the total crosssectional area of the fornix at the two levels was estimated both from photographs and from tracings made with the camera lucida on squared paper. In the sub-callosal part of the fornix, the average fibre density was 48 fibres per $100 \mu^{2}$; in the hypothalamus the density was greater, 74 fibres per $100 \mu^{2}$. The total cross-sectional area of the two fornix columns together, ventral to the corpus callosum, was $2,220,000 \mu^{2}$, both methods giving approximately similar figures. At the hypothalamic level only one fornix column was examined; its cross-sectional area was estimated to be $117,000 \mu^{2}$ by the camera lucida mensuration, and $147,000 \mu^{2}$ by the photographic technique. It is therefore estimated that each fornix column contains about 500,000 fibres before it enters the septum lucidum, and is reduced to not more than 110,000 fibres in the hypothalamus. The possible sources of error in this initial quantitative analysis are considerable, and more material is needed for confirmation of the results. The counts of fibre density were particularly difficult. However, these figures suggest that the precommissural component of the fornix is larger than the post-commissural, perhaps in a proportion as high as three or four to one. 


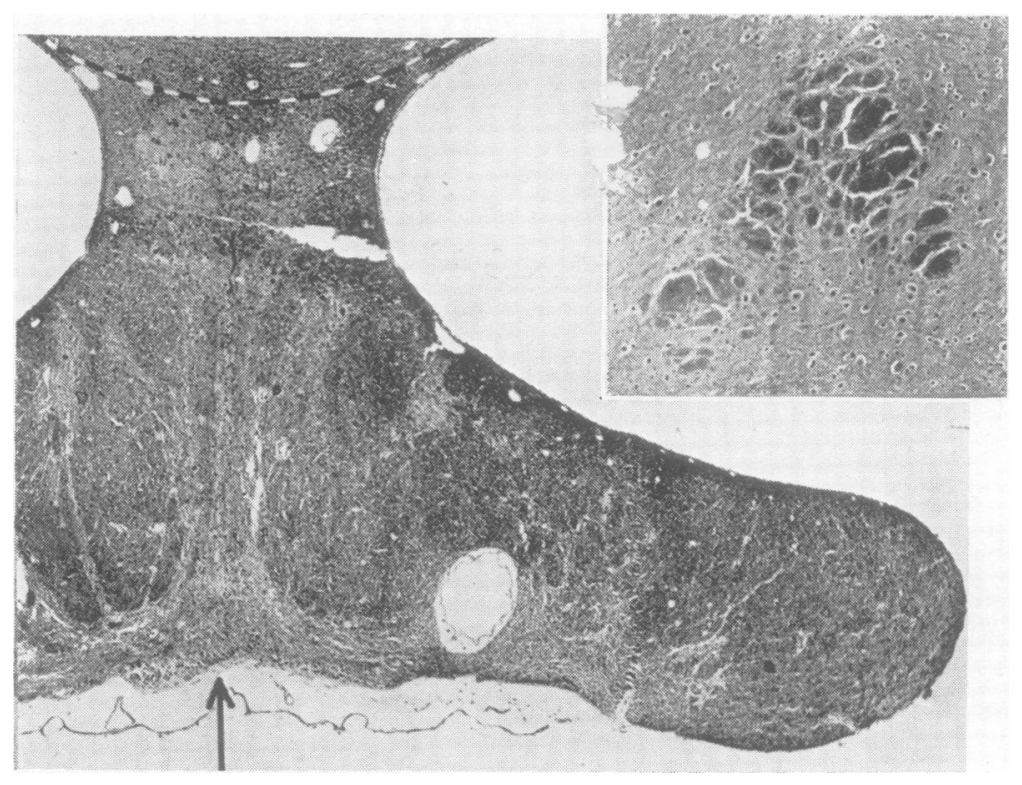

FIG. 1a.-Transverse section of ine fornix r olumn in the roof of the third ver.:- ile immediately behind the caudal extremity of the septum. The arrow indicates the median line between the two columns. The line of contact between the fornix column and the corpus callosum is marked by an interrupted line. Bodian stain $\times \mathbf{4 0}$.

FIG. 1b (inset).-Transverse section of the descending column of the fornix of the same brain, immediately above the mamillary body. Bodian stain $\times 40$. Note the marked contrast in cross-sectional area between the fornix in the roof of the third ventricle and the descending column.

Fibre Degeneration after Lesions in Fornix or Hippocampus.-The brains of seven monkeys have been examined. In three (Experiments 1, 5, and 7) lesions were made in the hippocampus of one side, and in four (Experiments 2, 3, 4, and 6) the subcallosal fornices were injured to varying extents. Additional information has been obtained from three brains in which ablations in the region of the temporal pole had been made by Adey and Meyer in a study (to be published) of its connexions.

The degeneration consequent on these lesions was studied with the ammoniacal silver method of Glees, slightly modified in this laboratory by M. Meyer (Adey and Meyer, in the press). Sections were usually cut with the freezing microtome at a thickness of 12 to $15 \mu$. It is well recognized that the evidences of degeneration provided by this method are liable to misinterpretation. In the present report fibres, whether pericellular and presumably terminal or preterminal, or grouped in tracts, are considered to be degenerating only when they are swollen and broken into beads or droplets (Figs. 2 and 3): variations in calibre without actual fragmentation have been disregarded. As occasional degenerating fibres are sometimes (though in the writer's experience rarely) seen in normal material, no deductions have been made from solitary fibres. Boutons terminaux have been accepted as evidence of terminal degeneration only when swollen and present in abnormally large numbers (e.g. in Fig. 4), for it is recognized (Meyer and Meyer, 1945 ; Glees, 1946) that isolated normal boutons terminaux are occasionally made visible by the Glees and similar methods. Wherever possible unaffected regions were examined as controls of the success of the staining, which is somewhat capricious, at least in the writer's hands. Adjacent sections stained by the methods of Weil and Nissl were employed to give accurate identification of tracts or nuclei studied with the silver technique.

The lesions were produced with the usual neurosurgical precautions. Their precise extent was deter- mined in various ways ; frozen sections were much less satisfactory for this purpose than serial sections of material embedded in low viscosity nitrocellulose, which, unlike paraffin wax, permits the use of the ammoniacal silver method.

Systematic behavioural and physiological studies were not made, as the survival times of the animals were necessarily brief. However, a few incidental observations of this kind are recorded.

The nomenclature of hypothalamic nuclei is that recommended by Rioch, Wislocki, and O'Leary (1940).

Fig. 5 illustrates schematically the fornix cyst.

FIG. 5.-Schematic representation of fornix system as seen from above; the diagram is numbered as a key to the subsequent figures. $1=$ hippocampus and dentate gyrus; $2=$ columns of the fornix ; $3=$ indusium griseum ; $4=$ medial mamillary nucleus $; 5=$ lateral mamillary nucleus; $6=$ ventromedial nucleus of the hypothalamus ; $7=$ septal nuclei,; $8=$ nucleus accumbens.

In these diagrams fibre tracts are delineated with thick lines and cell masses with thin lines. Terminal degeneration is indicated by dots and operative lesions in solid black. An interrogation mark has been placed where a structure has not been examined histologically.

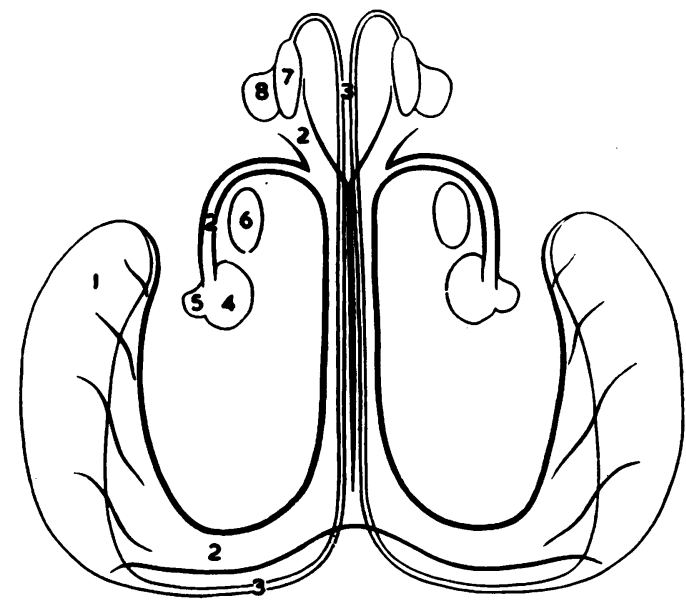




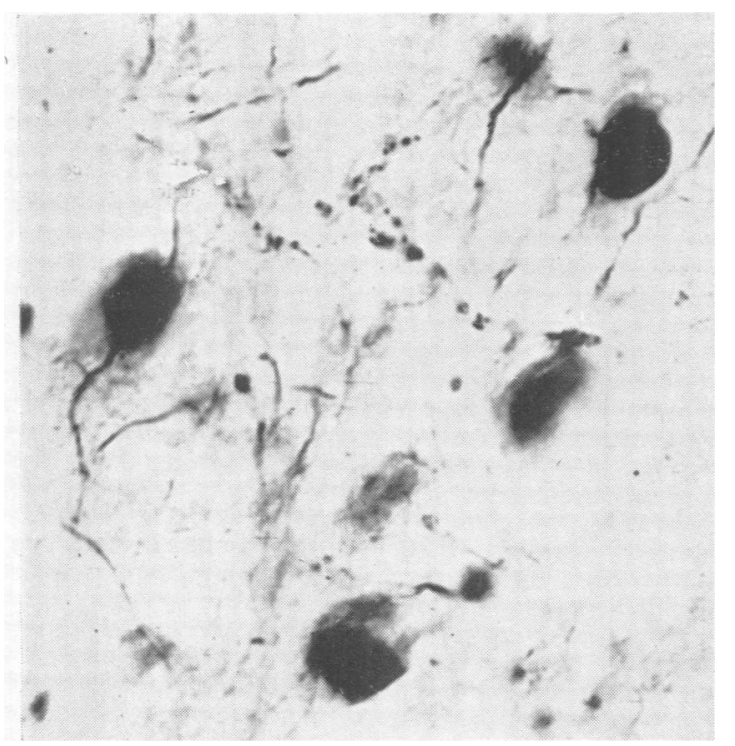

Fig. 2.-Pericellular fibre degeneration in the lateral septal nucleus (Experiment 6) Glees stain $\times 1600$.

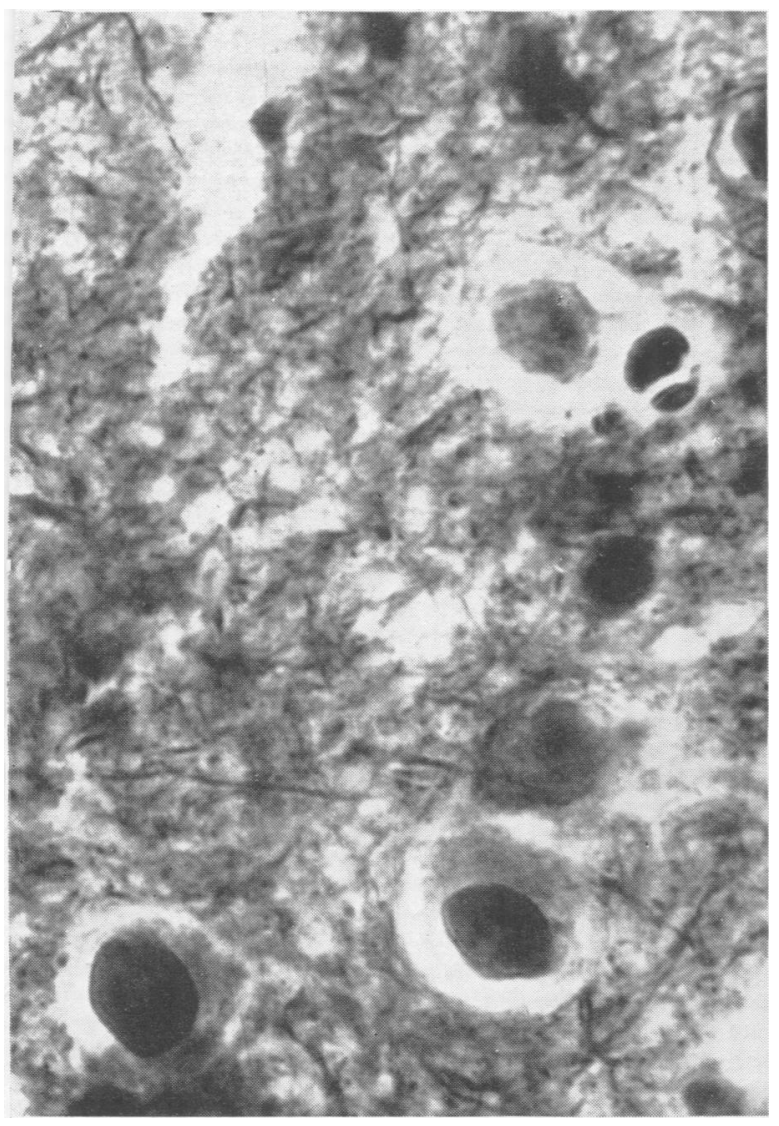

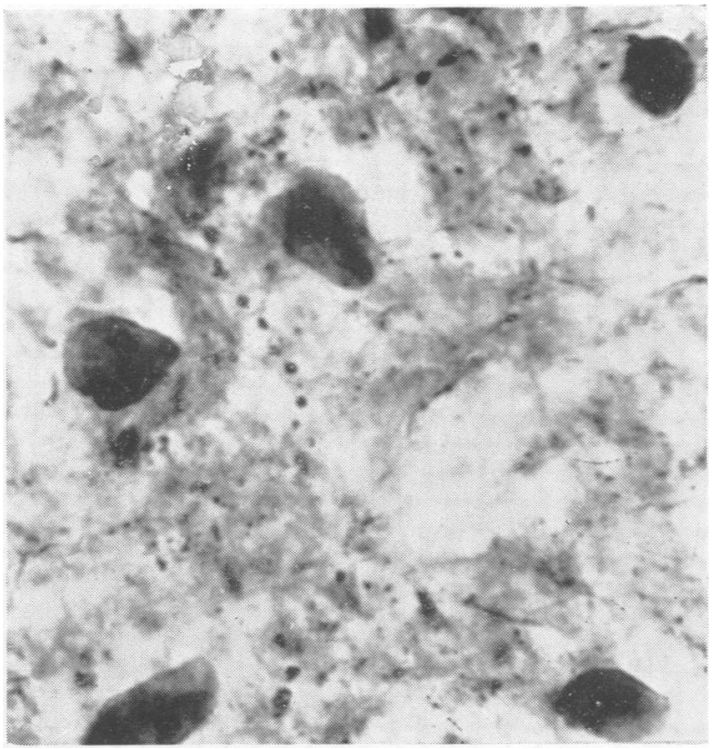

Fig. 3.-Pericellular fibre degeneration in the ventro-medial nucleus of the hypothalamus (Experiment 6). Glees stain $\times 1350$.

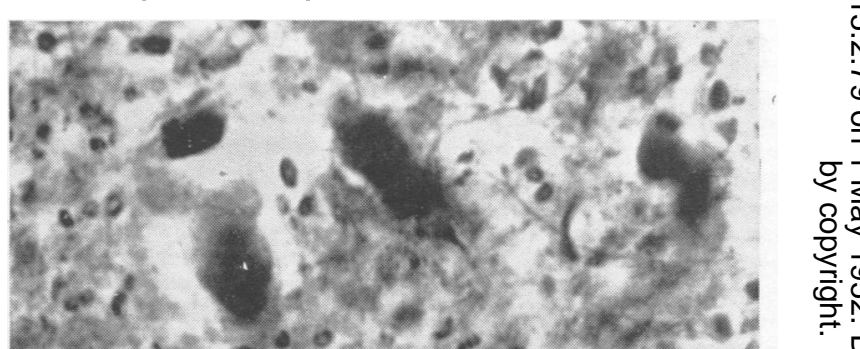

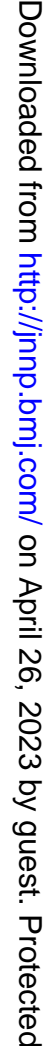




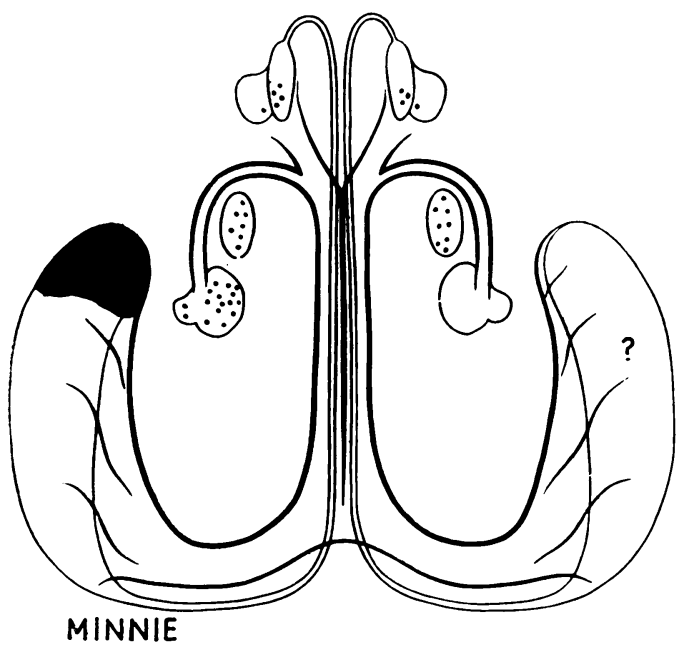

Fig. 6.-Injury to the anterior portion of the left hippocampus (Experiment 1).

\section{Experiments}

Experiment 1.-The subject, a Macaca mulatta, " Minnie," received at operation an injury of the anterior hippocampus (Fig. 6).

This monkey, whose brain was supplied by the courtesy of Dr. G. Weddell, was operated on in an attempt to sever the sensory root of the left trigeminal nerve. Access was obtained through the middle cranial fossa and, in retracting the temporal lobe, the uncus and the adjacent extremity of the hippocampus were damaged. Complications necessitated re-exposure of the trigeminal nerve root, and it is consequently uncertain precisely when the lesion was inflicted; it must have occurred between five and seven days before death. The animal was killed with nembutal and perfused with formol saline ; the brain was then removed and hardened in formaldehyde. This narcotic and this method of fixation have been employed in all subsequent experiments, with the exceptions of Nos. 3 and 4.

Examination.-The lesion extends obliquely across the ventral surface of the left temporal lobe in a rostral and medial direction. The amygdaloid complex has been injured, and the anterior extremity of the hippocampus is destroyed.

The hippocampi were embedded in gelatine and cut horizontally. Injury is seen to affect both the rostral tip of the left hippocampus and the dentate gyrus. Microscopical examination of the right hippocampus was unsatisfactory, due to a technical error in histological preparation.

The diencephalon was cut coronally and selected sections examined. In the mamillary region, very large numbers of boutons terminaux are present in the ventral part of the left medial mamillary nucleus. Some of them show unequivocal signs of degenera- tion, though there are no changes in neighbouring fibres. Caudally, about a quarter of the medial mamillary nucleus is so affected, but more rostrally only the most dorsal part is free from boutons (see Fig. 13). A very few boutons, showing in isolated instances suggestions of degeneration, are seen in the right medial mamillary nucleus, but they are probably of no significance. The left lateral mamillary nucleus contains a few swollen boutons terminaux ; as none are seen in the corresponding right nucleus, some importance attaches to them. The left nucleus intercalatus, in this brain a well defined cell mass, shows numerous degenerating boutons terminaux in its ventral part. A few degenerating fibres are also seen in the left supramamillary nucleus.

In the main part of the hypothalamus there is bilaterally massive pericellular fibre degeneration in the region of the ventromedial nucleus. No abnormality is seen in the perifornical nucleus, nor in the other hypothalamic nuclei. The fornix itself is not, at this early stage, abnormal in appearance.

There is unequivocai fibre degeneration in the most ventral part of the lateral septal nucleus, the n. septoventricularis of Brockhaus (1942). The identification of this nucleus, however, is uncertain; some of the degeneration appears to lie rather in the "fundus subventricularis medialis" of the same author, which corresponds with the most medial part of the nucleus accumbens of other authors. Both sides are affected. The rest of the n. accumbens, the tuberculum olfactorium, the nucleus of the diagonal band, and the other septal nuclei appear to be normal.

The habenulae and the brain-stem were not examined. No degeneration was seen in the indusium griseum or in the cingular gyrus.

Experiment 2. - The subject was a Macaca mulatta, MF94, and a transcallosal fornicotomy (Fig. 7) was performed.

The corpus callosum was exposed by a left parietal osteoplastic craniotomy; after retraction of the left cerebral hemisphere, the peri-callosal branches of the left anterior cerebral artery were coagulated and the corpus callosum incised sagittally. This incision was deepened until the third ventricle was visible; a rightangled steel hook, designed for the purpose, was then passed through the incision and rotated so as to lie in the coronal plane. It was then withdrawn in this position with the intention of severing the fibres of the fornix. The monkey was permitted to survive for eight days, during which time it exhibited a right spastic hemiplegia with intermittent seizures of a Jacksonian character. The brain was hardened in the manner described in the first experiment. 


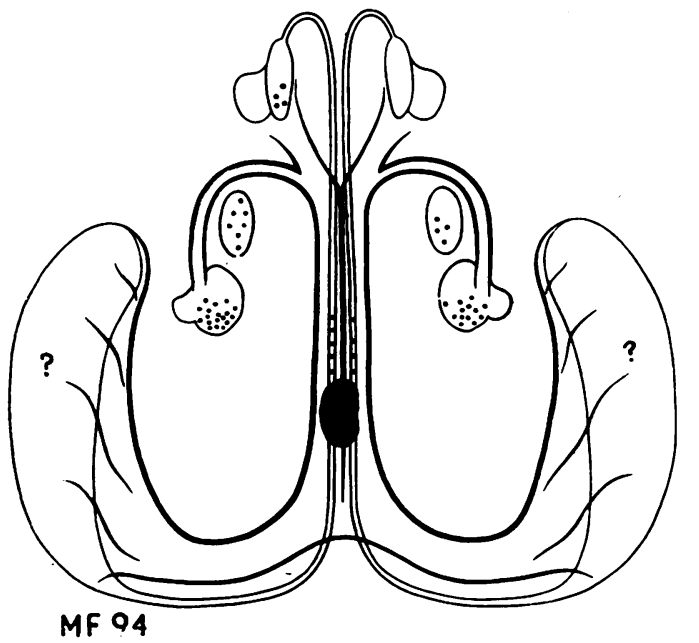

Fig. 7.-Bilateral partial section of the fornix column (Experiment 2).

Examination.-Gross inspection, supplemented by dissection under the binocular microscope, shows that rather more than half of the corpus callosum was injured ; there is fairly extensive softening of the medial surface of the left parietal lobe, involving the cingular gyrus. Both fornices have been injured, but neither completely severed. The diencephalon and anterior fornix were sectioned sagittally. Very numerous boutons terminaux are seen in the dorsal and caudal part of the medial mamillary nuclei (see Fig. 14); both sides are so affected, the left more extensively. The areas exhibiting this terminal degeneration are quite sharply demarcated, as is shown in the figure. No fibre degeneration is seen in the mamillary capsule. Of the minor mamillary nuclei, the nuclei intercalati, which are not easily defined in sagittal section, appear severely affected; the left supramamillary nucleus is normal and the left lateral mamillary nucleus probably so.

In the more rostral part of the hypothalamus there is definite fibre degeneration in a region corresponding with the rostral extremity of the ventromedial nucleus. The changes are more marked on the left side, but are present also on the right. The paraventricular, perifornical, and preoptic regions show no degeneration; the lateral hypothalamic area was not adequately examined.

The fornices show signs of local injury on their medial sides; retraction bulbs of Cajal are seen. However, the horizontal and descending portions of the fornix do not at this stage show signs of degeneration. Fibres of the pre-commissural component of the fornix lying ventral to the corpus callosum can be traced to the diagonal bands, but their further course is obscure.

There are a few degenerating fibres in the ventral part of the lateral (parvocellular) septal nucleus on the left side; the right appears to be unaffected. No degeneration is seen in the nucleus accumbens, the tuberculum olfactorium, or the nucleus of the diagonal band. Study of apparently undegenerated fibres of the fornix suggests, however, that this tract contributes to the diagonal band itself (c.f. Ariëns Kappers, Huber, and Crosby, 1936), though this cannot be determined with certainty. A few boutons terminaux are visible in the right medial habenular nucleus but, being neither numerous nor obviously swollen, are in themselves not conclusive evidence of terminal degeneration.

On both sides the striae Lancisii were severed in the splitting of the corpus callosum. Traced rostrally from the lesion, the medial striae exhibit severe degeneration, especially in the dorsal stratum ; the more ventral fibres are degenerated only in the immediate vicinity of the lesion. There are some boutons terminaux among these fibres, suggesting axo-dendritic synapses, and a few are also present among the neurons of the indusium griseum. The material does not allow the degenerated fibres to be followed as far as the genu; none of the fibres penetrating the corpus callosum are degenerated. The two hippocampi were embedded, sectioned in a plane transverse to their long axes, and stained with methylene blue; no retrograde changes could be detected.

Experiment 3.-The subject was a Macaca mulatta, MF98, and a transcallosal fornicotomy (Fig. 8) was performed.

The corpus callosum was exposed and incised in the manner described in the previous experiment, and the

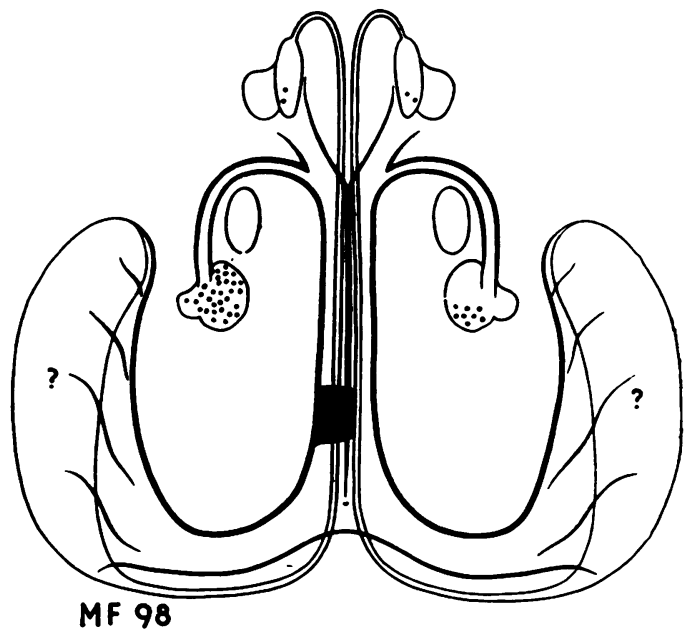

Fig. 8.-Section of the left fornix column and partial section of the right (Experiment 3). 
fornicotomy hook passed through the incision and rotated so as to lie athwart the left fornix. Diathermy current was then applied to the hook.

The monkey's condition after the operation was poor : it exhibited a right-sided hemiplegia and died six days after the operation. Necropsy showed hypostatic pneumonia and a severe nematode infestation of the colon; there was no intra-cranial infection or massive haemorrhage. The brain was hardened in formalin without perfusion.

Examination.-The brain was sliced in the coronal plane. The left fornix is seen to be cut in two places; it is grey and gelatinous in consistency. There are small haemorrhages and softening in the cingular gyrus, the pulvinar, and the dorsal thalamus; the internal capsule and basal ganglia are macroscopically intact. The diencephalic injury greatly reduces the value of this brain, and microscopic examination has been therefore limited to the site of injury, the mamillary nuclei, and the septal region.

A block containing the damaged fornices was embedded in paraffin and cut horizontally. Sections show the left fornix to be severely injured and probably wholly divided. There appears also to be injury in the contiguous, medial part of the right fornix ; but at this level the close approximation of the fornix columns makes distinction difficult.

Selected blocks from the diencephalon were sectioned coronally. Boutons terminaux are seen in both medial mamillary nuclei ; they are however less numerous and less strongly argyrophilic than those seen in other experiments, and their distribution is not easy to plot. On the left, the whole medial mamillary nucleus is apparently affected, but most severely in its dorsal portion. On the right, only a small area in the caudal and dorsal part contains boutons. A few boutons terminaux are seen in the left lateral mamillary nucleus but they are too few to constitute certain evidence of a projection to this structure. The nuclei intercalati are poorly developed, and the left nucleus cannot be defined; there is terminal degeneration in the right nucleus.

In the septal region there is very scanty bilateral fibre degeneration in the lateral septal nuclei, and possibly also in the immediately adjacent portion of the $n$. accumbens.

Experiment 4.-The subject was a Macaca mulatta, MF99, and the operation a transcallosal fornicotomy (Fig. 9).

The left fornix was injured with diathermy; the operative procedure was similar to that adopted in Experiment 3.

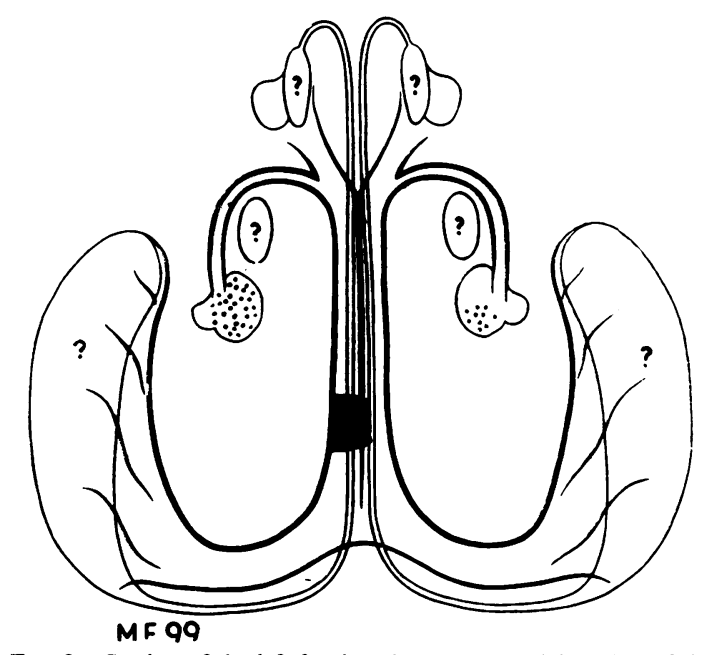

FIG. 9.-Section of the left fornix column and partial section of the right (Experiment 4).

Post-operatively the monkey showed signs of severe neurological affection, including a right-sided hemiplegia, a left oculomotor palsy, and a rhythmic tremor of the head, and on the fifth day it died. The brain was hardened without perfusion. Necropsy showed no extracranial disease of any importance.

Examination.-The brain shows no evidence of $\underset{2}{\overrightarrow{3}}$ infection or compression. However, there are $8 \underset{0}{2}$ numerous punctate haemorrhages in the left thalamus and basal ganglia, with displacement of the ventricular system to the right. The left fornix is apparently severed. As with Experiment 3, such extensive injury greatly complicates the interpretation of any degeneration, and only the lesion and the mamillary bodies have been examined microscopically.

A block containing the injured fornices was embedded in paraffin and cut horizontally. The left fornix has been totally divided; there is probably minor damage to the medial side of the right fornix. The lesion is therefore almost the duplicate of that in Experiment 3, and the distribution of terminal degeneration in the medial mamillary nuclei as seen in coronal sections (Fig. 13) is almost identical. Boutons terminaux in significant numbers are seen in both medial mamillary nuclei, in the whole volume of the left, but mainly in the dorsal part caudally of the right. As in Experiment 3 the distribution is not easily defined, probably because the shorter survival times give less obtrusive signs of terminal degeneration. The nuclei intercalati are small and spherical in this brain; both contain degenerating boutons. No certain evidence of terminal degeneration can be seen in the lateral mamillary nuclei. 


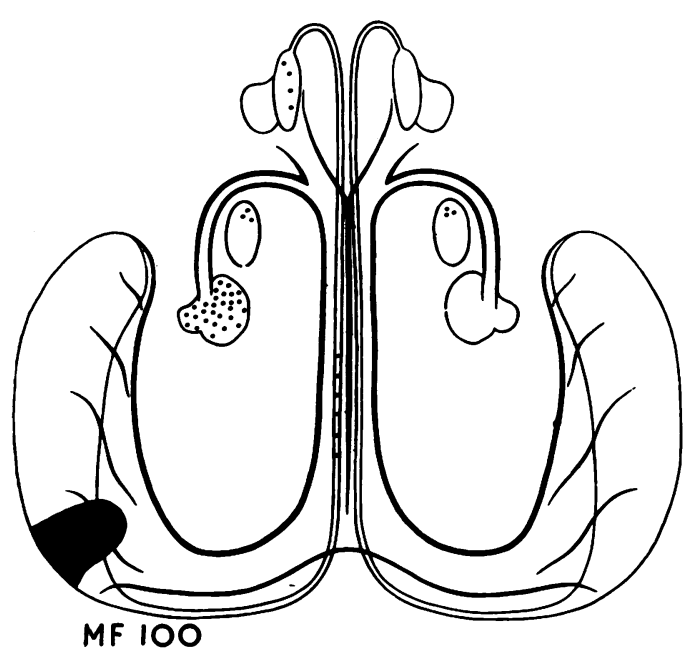

Fig. 10.-Injury to the posterior portion of the left hippocampus (Experiment 5).

Experiment 5.-A Macaca mulatta, MF100, at operation received an injury of the posterior hippocampus (see Fig. 10).

The posterior part of the left temporal lobe was exposed by the reflection of a small osteoplastic flap ; the middle temporal gyrus was incised with diathermy, and this incision was deepened with a fine-calibre sucker until the hippocampus was visible. A lesion was made by suction as far posteriorly as possible in the substance of the hippocampus. Post-operatively the monkey showed no signs of neurological injury; it showed, however, a definite change in behaviour, becoming less suspicious of its attendant. On the tenth day it was killed.

Examination.-External examination of the brain shows only the operational wound. A block containing the whole left hippocampus was embedded and sectioned in the transverse plane. Representative sections were stained with methylene blue and with iron haematoxylin. The lesion is seen to involve the caudal end of the hippocampus and dentate gyrus and the lateral half of the fimbria at the corresponding level. The most caudal extremity of the hippocampal complex has escaped injury ; rostral to the lesion the hippocampus and dentate gyrus appear normal, though the partial section of the fimbria must have severed many of their axons. The lesion does not involve the stria terminalis macroscopically; however, this tract shows some dubious fibre degeneration in sections stained by the Glees method. The major part of the entorhinal area escaped direct injury, but the temporo-ammonic tracts must have been interrupted in the hippocampal lesion.
The mamillary region was examined in sagittal section. The left medial mamillary nucleus contains very numerous boutons terminaux; many of them show obvious signs of degeneration. Their distribution is shown in Fig. 14. They are accompanied by fine degenerating preterminal fibres, not seen in previous experiments. The left nucleus intercalatus contains a few degenerating fibres. The left lateral mamillary nucleus contains numerous degenerating terminals. The right medial mamillary nucleus exhibits a very small number of boutons terminaux ; though these are occasionally seen in normal preparations, the possibility of a projection by a few fornix fibres to the contralateral medial mamillary nucleus cannot be definitely excluded. In the hypothalamus there is bilateral pericellular degeneration in a region corresponding to the junction of the ventromedial nucleus with the anterior hypothalamic area; it is probable that both these cell groups are affected. The perifornical region and the fornices themselves show no detectable change. Attempts to trace fornix fibres into the midbrain were unsuccessful, but the existence of such fibres cannot be excluded.

In the septal region (examined in sagittal section) terminal degeneration is seen in the dorsal part of the left lateral septal nucleus. Degenerating fibres are also seen passing horizontally through the septal nuclei ; the appearances suggest that these fibres are going to the diagonal band, but this cannot be certainly determined. No degeneration is seen in the nucleus accumbens, the tuberculum olfactorium, the nucleus of the diagonal band, or the right septal nuclei.

Sections of the habenular nuclei show no degeneration but the sections taken in this region are poor in quality.

The striae Lancisii and indusium griseum were examined, also in sagittal section : the left medial stria shows degenerating fibres running dorsal to the neurons of the indusium; a few degenerating boutons de passage are seen between the fibres. The right stria appears normal.

The right hippocampal region was sectioned horizontally. Careful examination of the hippocampus and dentate gyrus reveals no abnormality ; portions of subiculum, entorhinal area, and of the amygdaloid complex were also examined and no degeneration seen.

Experiment 6.- The subject was a Macaca mulatta, MF101, and the operation transcallosal fornicotomy (Fig. 11).

The splenium of the corpus callosum was exposed by the elevation of a large right occipito-parietal osteo- 


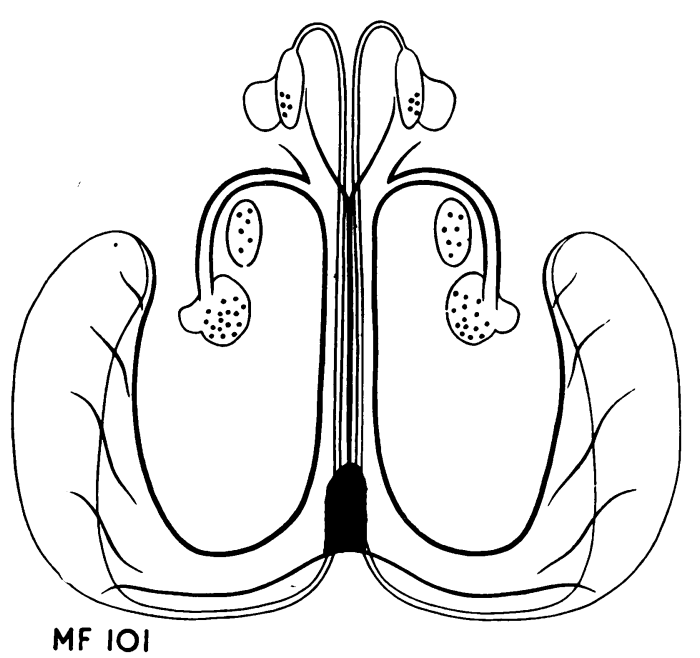

FIG. 11.-Bilateral partial section of the fornix columns and the psalterium (Experiment 6).

plastic flap. The pericallosal branches of the anterior cerebral vessels were coagulated and the splenium completely divided in the median line. It was hoped by this manoeuvre to sever the psalterium. The fornices were seen and lesions made in the right fornix without the use of diathermy. Post-operatively the monkey's condition was excellent and, as in the previous experiment, it was definitely more docile than before the craniotomy. Thirteen days after the operation the monkey was killed.

Examination.-Externally the brain shows no damage or disease, other than the wound in the corpus callosum.

A block including the damaged fornix and the surrounding structures was embedded and sectioned horizontally. There are infarctions in both cingular gyri, due no doubt to the coagulation of the cortical branches of the anterior cerebral arteries. The corpus callosum has been divided for a distance of $1-1 \frac{1}{2} \mathrm{~cm}$. The adjacent, medial sides of both fornices have been injured, the left more severely. The psalterium has presumably been divided, but this commissure cannot be identified. There is no macroscopic evidence of injury to the striae terminales, but sections of these tracts, cut parallel to their fibres, show possible signs of degeneration in the right stria.

The mamillary nuclei were examined in coronal section. Both medial mamillary nuclei contain degenerating boutons terminaux in their caudal and dorsal portions (see Fig. 13); the area involved is considerably more extensive on the left side. The supramamillary nuclei are not affected. A few boutons are seen in both lateral mamillary nuclei.
The left nucleus intercalatus, which is poorly demarcated, contains degenerating boutons; the right nucleus could not be defined at all.

In the hypothalamus, sectioned coronally, there is exceedingly severe pericellular fibre degeneration in both ventromedial nuclei. The remainder of the hypothalamus, including the perifornical nuclei, appears normal.

In the septal region, also cut coronally, there are many degenerating fibres in the ventral parts of both lateral septal nuclei. The area affected is circumscribed, and probably corresponds with Brockhaus' (1942) " n. septo-ventricularis". The nucleus accumbens and the tuberculum olfactorium appear to be unaffected.

The habenulae were cut coronally. Very massive terminal degeneration, including some bizarre endings, is present in both medial habenular nuclei, but this is almost certainly due to direct injury inflicted when the fornices were divided immediately above the habenulae. The right hippocampal complex was embedded, cut in a plane transverse to its long axis, and stained with thionin. No chromatolysis is seen in the cells of hippocampus, subiculum, or entorhinal area. The left hippocampal complex was cut in the same plane, and selected sections stained with the method of Glees. No degeneration is seen in the alveus, hippocampus, dentate gyrus, subiculum, or entorhinal area.

Experiment 7.-The subject, a Cercopithecus sabaeus, MF102, received at operation an injury of the central part of the hippocampus (Fig. 12).

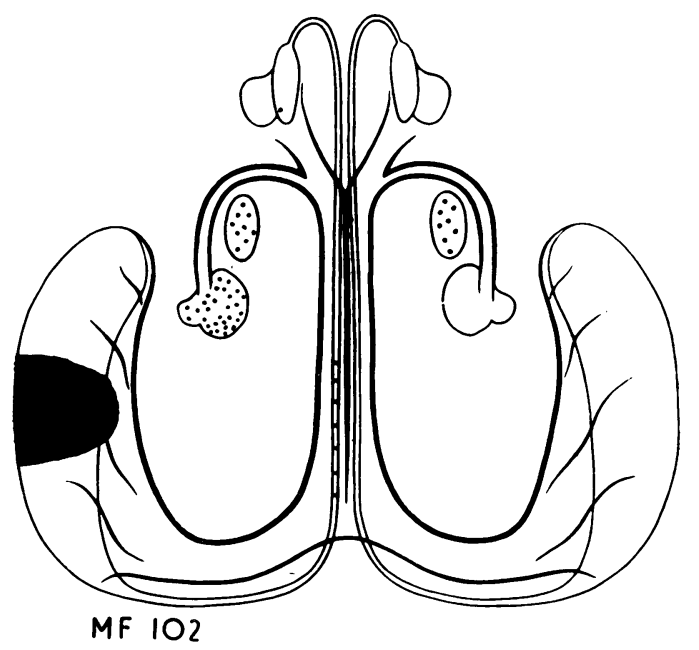

Fig. 12.-Injury to the central portion of the left hippocampus (Experiment 7). 
The left temporal lobe was exposed through an enlarged trephine hole, and the middle temporal gyrus incised with diathermy. The incision was deepened, in a medial and ventral direction, until it was estimated that the hippocampus had been traversed. Haemorrhage prevented identification of the hippocampus and lateral ventricle. Post-operatively, there were no obvious neurological defects ; however, the monkey, which had previously exhibited a morose and even spiteful disposition, was decidedly more docile. Seven days after the operation, the monkey was killed.

Examination.-Externally, the brain showed no disease or injury other than the operation wound. A block containing the left hippocampus was embedded and cut sagittally. Sections show the lesion as a cavity about $1 \mathrm{~cm}$. in diameter penetrating the temporal lobe from its lateral surface medially as far as the hippocampus. The central third of the hippocampal complex is totally destroyed; the fimbria is injured but numerous fibres in it appear to have escaped destruction. The stria terminalis has been severed; the amygdaloid nuclei are intact.

The brain-stem and posterior diencephalon were cut, as a single block, in the sagittal plane. The left medial mamillary nucleus contains numerous degenerating boutons terminaux, especially caudally; the distribution is shown in Fig. 14. The right medial mamillary nucleus contains no significant evidence of terminal degeneration. The left lateral mamillary nucleus exhibits a small but abnormal number of boutons terminaux. The left nucleus intercalatus is large but ill-defined; it contains many degenerating terminals.

The pontine and tegmental nuclei, and especially the ventral tegmental nucleus of Tsai (1925) and the reticular formation, have been carefully examined ; their anatomy is difficult to establish in sagittal section. No terminal degeneration is seen, but it is possible that minor changes have escaped notice. An unsuccessful attempt was made to trace undegenerated fibres from the fornix to the tegmentum; if such fibres do exist, they must be diffuse and not, as in the rabbit, a compact bundle.

In the hypothalamus the ventromedial nuclei are filled with most severe pericellular degeneration; other nuclei appear normal.

The septal region, horizontal fornices, and corpus callosum were sectioned sagittally. The septal nuclei show unexpectedly little terminal degeneration : careful search reveals only a few fragmented fibres in the ventral part of the left lateral septal nucleus.

In the left medial stria Lancisii there is wellmarked degeneration of long fibres and of boutons de passage. There is also some terminal degeneration around the cells of the left indusium griseum. No abnormality is seen on the right side. The habenular nuclei, examined in sagittal section, appear normal.

The right hippocampus was sectioned transversely to its long axis. No degeneration is seen in dentate gyrus, hippocampus, or subiculum ; the entorhinal area was only partially examined, but appears unaffected.

\section{Supplementary Experiments}

The following experiments were performed by Adey and Meyer in the course of a study (to be published) of the connexions of the temporal pole. By the courtesy of these authors, they are reported here in so far as they concern the present study.

Experiment 8. -The subject was̀ a Cercopithecus s $_{\mathrm{i}}$ sabaeus, M85.

In this brain there is a massive lesion involving. almost the whole of the amygdaloid complex and extending into the entorhinal area and the anterior: tip of the hippocampus itself.

Boutons terminaux are present in the most ventral part of the ipsilateral medial mamillary body, occupying an area rather more extensive rostrally. than caudally. The area affected resembles that: described in Experiment 1 very closely, but is: smaller in the dorso-ventral dimension.

Experiment 9.-The subject was a Cercopithecus sabaeus, M91.

In this case the lesion is restricted to the entorhinal area and amygdaloid complex. The hippocampal formation is mainly intact, save for a small lesion with inflammatory reaction in the alveus anteriorly. The ipsilateral medial mamillary nucleus, examined in coronal section, shows a very small but unquestionably significant number of boutons terminaux in its most rostral and ventral part.

Experiment 10.-The subject was a Cercopithecus sabaeus, M86.

In this brain the lesion involves the amygdaloidcomplex, with the exception of its medial and cortical nuclei and the nucleus of the lateral olfactory tract ; in consequence, the stria terminalis has' degenerated. A portion of the superior temporal gyrus and the prepiriform cortex have been destroyed, but the hippocampus is uninjured. No terminal degeneration is seen in those parts of the medial mamillary nuclei which were affected in the two preceding experiments. 


\section{Discussion}

The present study was planned to give a comprehensive picture of the efferent projection of the hippocampus in the monkey, with special reference to speculations upon its possible functions. The aim has been only partially successful. There remain important lacunae in the data which express the limitations of the ammoniacal silver method, at least in the writer's hands, and the technical diffi- culty of interrupting the fornices without coincidental damage to adjacent structures. However, certain conclusions may be stated with some confidence. The hippocampus projects to the medial mamillary nucleus of the same side. The fibres terminate in all parts of the medial mamillary nucleus and there appears to be fairly precise spatial correlation between their origins and terminations. This is well seen in the coronal and sagittal sections of mamillary
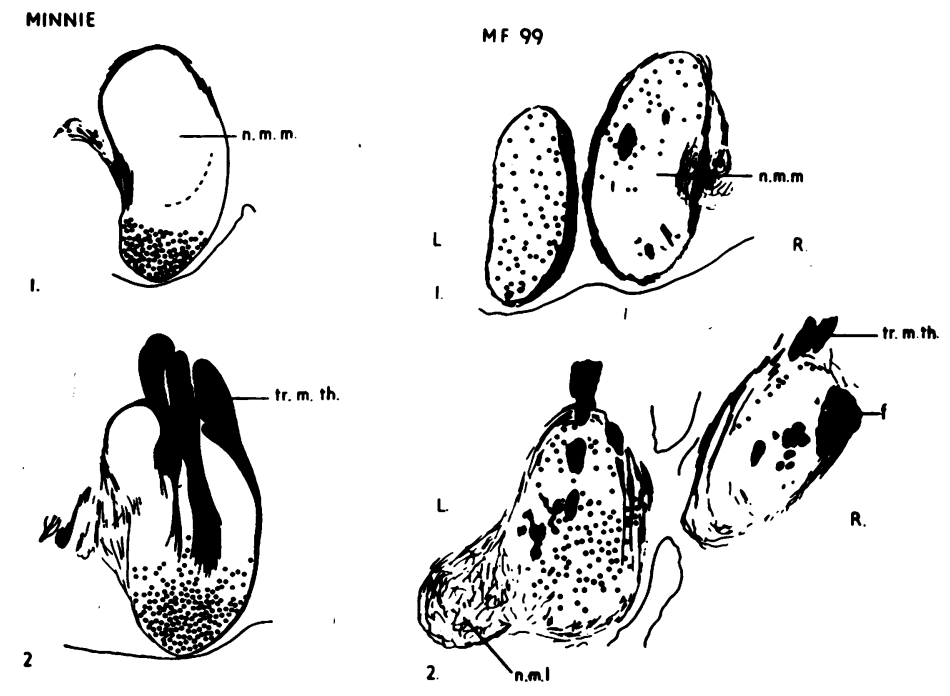

MF 101
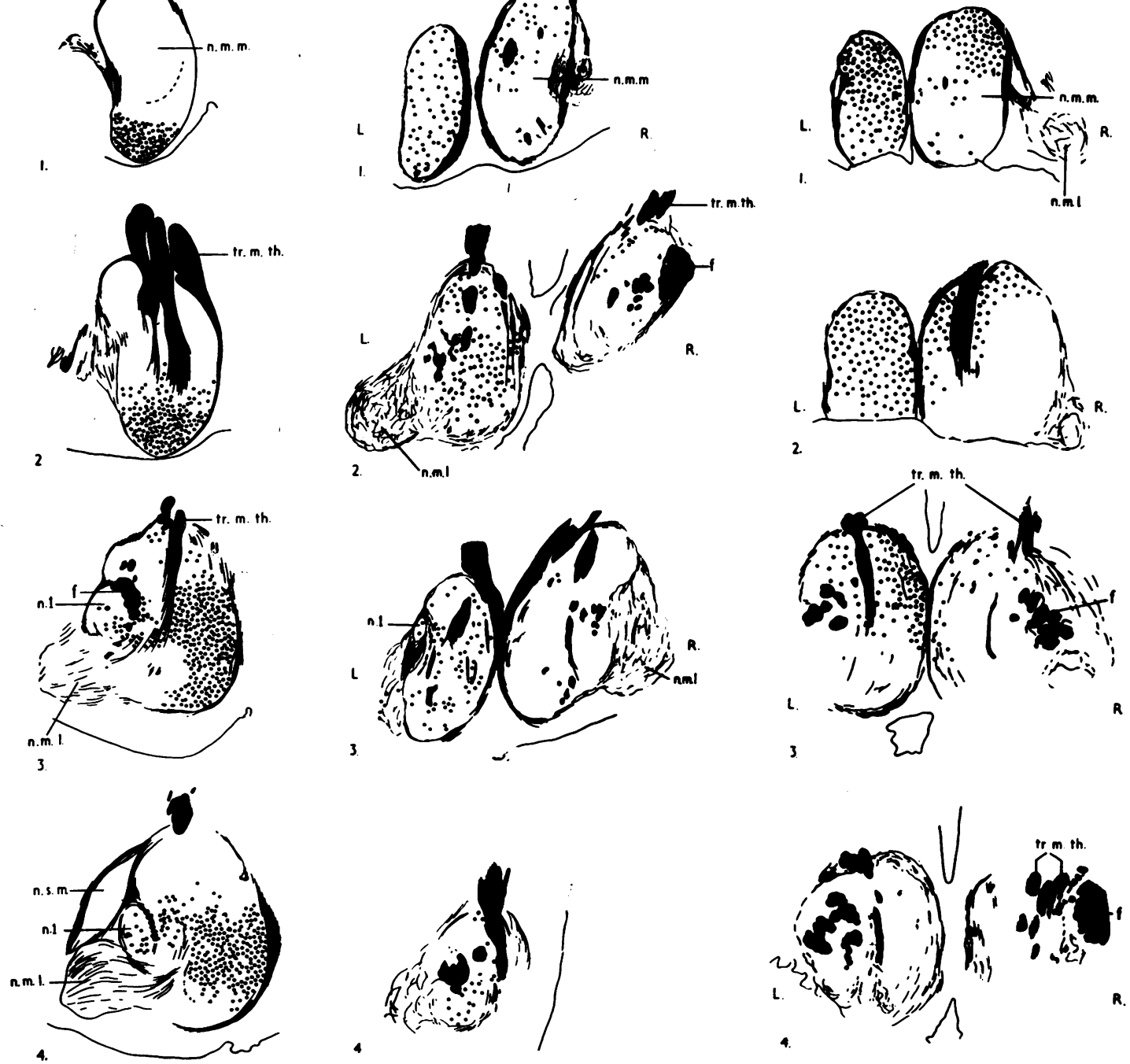

FIG. 13. - The mamillary bodies in Experiment 1 (Minnie, left side only), Experiment 3 (MF99), and Experiment 6 (MF101) $\times 12$.

The drawings are made with the camera lucida from coronal sections; the presence of terminal degeneration is indicated by dots whose density corresponds approximately with that of the terminal degeneration.

tr.m.th. $=$ mamillo-thalamic tract $; f=$ fornix $;$ n.m.m. $=$ medial mamillary nucleus; $n . i .=$ nucleus intercalatus; n.m.l. $=$ lateral mamillary nucleus. 

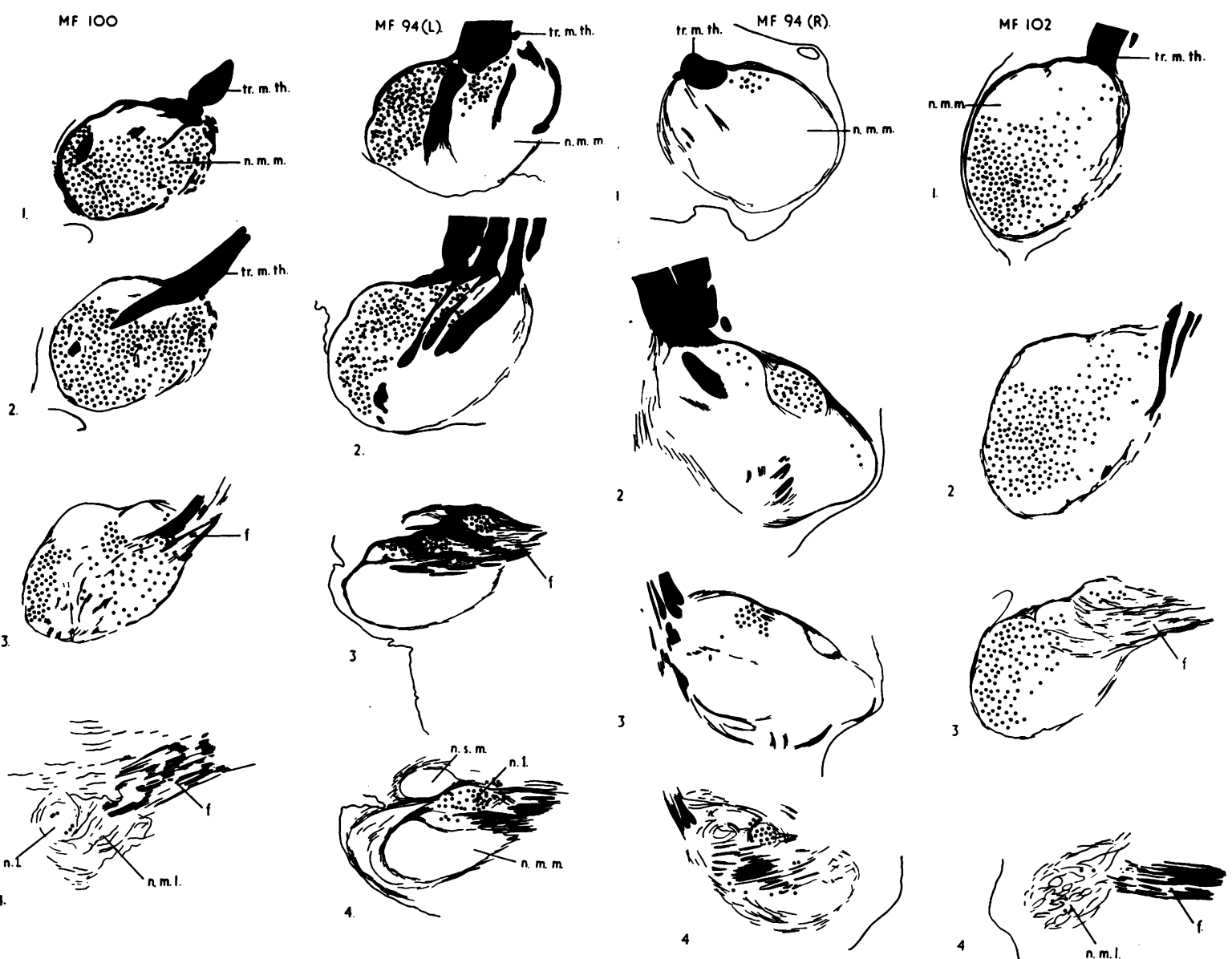

FIG. 14.-The mamillary bodies in Experiment 2, (MF94, both right and left sides), Experiment 5 (MF100, left side only), and Experiment 7 (MF102, left side only) $\times 12$.

The drawings are made with the camera lucida from sagittal sections.

bodies illustrated in Figs. 13 and 14, contrasted with the diagrams which show the various lesions. For the purposes of description, the medial mamillary nucleus may be regarded as an oval mass, at the rostral and dorsal pole of which the mamillothalamic tract emerges; the long axis is thus disposed obliquely to the horizontal plane. The distribution of terminals indicates a stratification in layers parallel to this long axis. Thus the anterior extremity of the hippocampus projects to the ventral and rostral level (Experiment 1 and the Supplementary Experiment 8), and the central part of the hippocampus projects to the axial zone (Experiments 5 and 7). The most caudal and dorsal stratum, which is unaffected in Experiments 1, 5, 7, and 8 presumably receives fibres from the most caudal portion of the hippocampus. An alternative explanation of the absence of degeneration in these experiments is that this unaffected level receives fibres of supracallosal origin, the fornix longus. However, study of two brains with lesions in the cingulate gyrus gives no evidence to support this hypothesis. Experiments 2, 3, 4, and 6 suggest that there is an orderly marshalling of fibres in the fornix also, the most medial fibres projecting to the dorso-caudal stratum of the medial mamillary nucleus. Gross dissections demonstrate that the posterior fibres of the alveus run medially in the fornix, and the anterior fibres laterally. The nucleus intercalatus also receives a fornix contribution. The lateral mamillary nucleus appears to receive a few fornix fibres, but except in Experiment 6 the number of terminals was not large. All previous investigators have described fornix fibres proceeding to 
the mamillary body, but the strikingly orderly arrangement of terminals seen in the monkey has not been reported in lower mammals. Moreover, Ramon y Cajal (1909), whose Golgi preparations were made on rodent and cat brains, states that the component of the fornix which ends in the mamillary region is smaller than that passing caudally to the tegmentum; this is certainly not the case in the monkey. Several earlier workers have described a more considerable projection to the lateral mamillary nucleus than is demonstrable in the monkey (e.g. Gerebtzoff, 1941-42); however, this may be a consequence of the use of the Marchi method which does not permit certain identification of the ultimate destiny of fine fibres.

No evidence for a supramamillary decussation was obtained; this decussation is described in the rabbit by Vogt (1898) and by Edinger and Wallenberg (1902). The last named authors observe that there appears to be a difference in the proportion of decussating fibres between different varieties of rabbit, which is of significance in view of the inability of Gerebtzoff (1941-42) and Sprague and Meyer (1950) to find any decussation in their preparations of rabbit brains. A tegmental overshoot of the fornix was not demonstrated. While the possibility that a few fornix fibres pass into the tegmentum cannot be excluded, it seems certain that there is no considerable bundle such as is seen in the rabbit (Edinger and Wallenberg, 1902 ; Yamagata, 1929 ; Gerebtzoff, 1941-42). Functionally, of course, the mamillo-tegmental tract provides an alternative route whereby fornix impulses may be relayed to the tegmentum.

Experiments 6 and 8 demonstrate unequivocally that the hippocampus is intimately connected with the striae Lancisii and indusium griseum, as might be expected from the comparative anatomy of these structures.

The precommissural connexions of the fornix have been much more difficult to determine. Study of normal material shows that a large component of fornix fibres extends rostral to the anterior commissure, and the fibre counts reported in this paper suggest that this component is considerably larger than the contribution to the mamillary nuclei. Yet in our experiments terminal degeneration has been seen only in the lateral septal nucleus and is here by no means extensive. It is of course probable that some of the fibres in the precommissural fornix are septal efferents, as described by Gerebtzoff (194142), Morin (1950), Sprague and Meyer (1950), and Stoll, Ajmone-Marsan, and Jasper (1951) ; Allen (1944), however, denies their existence. Also to be considered is the possible presence of a forward extension of commissural fibres such as Tumbelaka (1915) identified both in the brain of an acallosal monkey and in normal material. It yet remains likely that the precommissural fornix includes hippocampal efferents not made evident in this study. These fibres may perhaps be distributed beyond the septum to more remote areas by way of the diagonal band and medial forebrain bundle.

The position regarding a hippocampal projection to the main part of the hypothalamus is also unsatisfactory. Terminal degeneration was seen in the ventromedial nucleus of the hypothalamus in all experiments in which this region was examined, but it is known (Adey and Meyer, personal communication) that this nucleus receives a projection from the amygdaloid complex, and damage either to the latter or to its efferent tract, the stria terminalis, can be excluded in none of these experiments. However, from a careful consideration of the experimental evidence, it seems probable that the fornix does send a small contribution to the region of the ventromedial nucleus, though positive confirmation of this is needed. The absence of changes in the perifornical nuclei is unexpected and of some interest; Sprague and Meyer (1950) observed terminal degeneration in this region in the rabbit.

Negative evidence is presented with some reluctance, as it is only too easy to miss minor degrees of terminal degeneration. But the absence of terminal degeneration in the habenular nuclei is probably of significance. Several authorities, notably Gerebtzoff (1941-42), have described fornix fibres ending in the habenular nuclei and even in the pineal body, but as Vogt (1898) and Sprague and Meyer (1950) have pointed out, it is difficult to section the fornix without concurrent injury to structures known to project toward the habenular region, such as the stria medullaris and the fasciculus septo-thalamicus. Experiments 5, 6, and 7 strongly suggest that the hippocampal commissure is an unimportant structure in the monkey, and this is in accord with the studies of normal material made by Beevor (1891). It had been hoped that a study of retrograde degeneration would decide whether cells other than the hippocampal pyramids and the cells of the indusium griseum contribute axons to the fornix, an important question which has attracted surprisingly little attention. However, in Experiments 2 and 6 no chromatolytic changes were evident, probably because of the short survival times. Experiments 9 and 10 suggest that the amygdaloid complex sends no fibres to the mamillary nuclei. The entorhinal area may possibly do so, but this is considered unlikely; the small number of degenerating terminals seen in Experiment 9 can be accounted for by 
the accompanying lesion in the alveus. It may be concluded, therefore, that the commonly accepted view is correct, that the fibres of the fornix destined to the mamillary body are of strictly hippocampal origin. Whether this is true of those fibres which terminate in the septum, however, is an open question. In the recent electroneuronographic study by Stoll, Ajmone-Marsan, and Jasper (1951), both afferent and efferent fibres were found connecting septal nuclei with " the tip of the temporal lobe ", and travelling in the fornix. Cytoarchitectural data are not included in this paper, but presumably these fibres are of neocortical, i.e., extra-hippocampal, origin.

The experiments reported here, and the study of previous examinations of the efferent projection of the hippocampus, do not permit of formulating functional hypotheses. The functions of the mamillary nuclei are still obscure. Their most important projection is to the anterior thalamic nuclei, which in turn project to the cingulate cortex, and it may well be of significance that the primate hippocampus is related so intimately, and with such a sharp spatial localization, to the medial mamillary nucleus. Lower mammals do not show such an arrangement (Sprague and Meyer, 1950).

Papez (1937) presented the interesting hypothesis " that the hypothalamus, the anterior thalamic nuclei, the gyrus cinguli, the hippocampus and their interconnexions constitute a harmonious mechanism which may elaborate the functions of central emotion, as well as participate in emotional expression". Animal experiments have demonstrated profound behavioural changes after bilateral lesions in the temporal lobes and even after bilateral fornicotomy (Spiegel, Miller, and Oppenheimer, 1940). Various speculative interpretations of these data have been made, and perhaps the most extreme is MacLean's (1949) hypothesis that the structures commonly termed the rhinencephalon constitute a "visceral brain", associating all forms of sensation with autonomic and emotional activity. However, the hypothesis of direct and specific participation by the hippocampus and fornix system in emotional activities involves a large element of speculation. The results of animal experimentation are by no means in accord and, as the records of the experiments reported in this paper demonstrate, it is extremely difficult to injure the hippocampal system without concurrent injury to the amygdaloid complex and stria terminalis. The absence of gross mental changes after section of the human fornices (Dott, 1938 ; Cairns, 1951) and the apparently normal mentality of a man whose brain shows virtual absence of the fornices due to malformation
(Nathan and Smith, 1950), suggest that the hippocampal system subserves some more subtle function. It may be that this function is in the nature of a nonspecific activation or regulation of cortical mechanisms, and also of sub-cortical centres, particularly in animals lower in the phylogenetic scale. In this view, the behavioural changes which have been reported in experimental animals following lesions involving the hippocampal system may express a disorganization of normal responses, rather than the interruption of a specific " emotional pathway". One may postulate that in the human brain other mechanisms can compensate for the defect of hippocampal activity.

Morphology of Terminal Degeneration.-In conclusion, some comments on the morphology of degeneration in this system may be of value. The fibres in the fornix of the monkey are extremely slow to degenerate ; even 10 days after section, the ammoniacal silver method shows no great alteration in the appearance of its fibres. Degenerating boutons terminaux appear however by the fifth day and are still well seen on the thirteenth. It is noteworthy that the fornix appears to terminate solely by boutons in the medial mamillary nucleus, while the degeneration seen in the ventromedial nucleus of the hypothalamus and in the septal region is of the pericellular type. It seems very probable that there is a physiological distinction between these types of ending ; the first is perhaps adapted for transmission to a small local group of cells, the second for a more diffuse type of stimulation. It is interesting to note that Sprague and Meyer (1950) found relatively fewer boutons in the medial mamillary nucleus of the rabbit.

\section{Summary}

In the monkey the hippocampus projects to the ipsilateral medial mamillary nucleus, and to a less extent to the lateral mamillary nucleus and the nucleus intercalatus. It is probable that all the fornix fibres destined to the mamillary body originate from the hippocampus, and there is a definite spatial correlation between the cells of origin in the hippocampus and the terminal boutons in the medial mamillary nucleus. It is suggested that this is the most important projection of the primate hippocampus.

The precommissural component of the fornix in the monkey is large, but the destination of these fibres has not been determined with the exception of a small number ending in the lateral septal nucleus. It is probable that many of these fibres are not of hippocampal origin. 
The hippocampal commissure is greatly reduced in the primate brain.

It is probable that the primate fornix sends fibres to the ventromedial nucleus of the hypothalamus, but no evidence to support a projection to the habenulae or the tegmentum has been obtained.

The hippocampus projects to the indusium griseum and supracallosal striae.

The fornix fibres end as boutons terminaux in the medial mamillary body, but as pericellular fibre plexuses in the septal region.

I wish to thank Professor W. E. Le Gros Clark, who planned this investigation and has assisted at all stages of its preparation, Mrs. S. Nevin for advice in the interpretation of the sections, and Dr. A. C. Allison for advice on the technique of counting fibres. I wish also to thank Miss Christine Court for the drawings, Mr. L. D. Parke, who prepared much of the histological material, and Mr. F. M. Blackwell for the photomicrographs.

\section{REFERENCES}

Adey, W. R. (1951). Brain, 74, 233.

, and Meyer, M. (1952). J. Anat., Lond., In the press.

Allen, W. F. (1944). J. comp. Neurol., 80, 283. (1948). Ibid., 88, 425.

Allison, A. C., and Chacko, L. (1949). J. roy. Micr. Soc., $69,18$.

Beevor, C. E. (1891). Philos. Trans., B, 182, 135.

Brockhaus, H. (1942). J. Psychol. Neurol., Lpz., 51, 1. Brodal, A. (1947). Brain, 70, 179.

Cairns, H., and Mosberg, .W. H. (1951). Surg. Gynec. Obstet., 92, 545.

Clark, W. E. Le Gros (1932). Philos. Trans., B., 222, 1.

Dejerine, M., and Dejerine, J. (1897). C. R. Soc. Biol., Paris, Ser, 10, 4, 587.
Dott, N. M. (1938). In Clark, W. E. Le Gros, Beattie, J., Riddoch, G., and Dott, N. M., "The Hypothalamus", pp. 131-185. Edinburgh.

Edinger, L., and Wallenberg, A. (1902). Arch. Psychiat. Nervenkr., 35, 1.

Fox, C. A."(1943). J. comp. Neurol., 79, 277.

Gerebtzoff, M. A. (1941-42). J. belge Neurol. Psychiat., 41-42, p. 199.

Glees, P. (1946). J. Neuropath. exp. Neurol., 5, 54.

Gudden, B. von (1881). Arch. Psychiat. Nervenkr., $11,428$.

Kappers, C. U. Ariëns, Huber, G. C., and Crosby, E. C. (1936). The Comparative Anatomy of the Nervous System of Vertebrates, including Man. New York, Macmillan.

MačLean, P. D. (1949). Psychosom. Med., 11, 338.

Meyer, A., and Meyer, M. (1945). J. Anat., Lond., 79, 180.

Morin, F. (1950). J. comp. Neurol., 92, 193.

Nathan, P. W., and Smith, M. C. (1950). Journal of Neurology, Neurosurgery and Psychiatry, 13, 191.

Papez, J. W. (1937). Arch. Neur. Psychiat., Chicago, 38, 725.

Pribram, K. H., Lennox,M. A., and Dunsmore, R. H. (1950). J. Neurophysiol., 13, 127.

Probst, M. (1901). Arch. Anat. Physiol., Anat. Abt., Lpz., 338

Ramon y Cajal, S. (1909). "Histologie du système nerveux de l'homme et des vertébrés," trans. Azoulay, L. Paris.

Rioch, D. McK., Wislocki, G. B., and O'Leary, J. L. (1940). In "The Hypothalamus." Res. Publ. Ass. nerv. ment. Dis., 20, 3.

Spiegel, E. A., Miller, H. R., and Oppenheimer, H. J. (1940). J. Neurophysiol., 3, 538.

Sprague, J. M., and Meyer, M. (1950). J. Anat., Lond., 84, 354.

Stoll, J., Ajmone-Marsan, C., and Jasper, H. H. (1951) J. Neurophysiol., 14, 305.

Tsai, C. (1925). J. comp. Neurol., 39, 173.

Tumbelaka, R. (1915). Folia neuro-biol., 9, 1.

Vogt, O. (1898). C. R. Soc. Biol., Paris, Ser. 10, 5, 206.

Yamagata, K. (1927). Okayama Igakkai Zasshi, 39, 1184. (German summary, p. 1200).

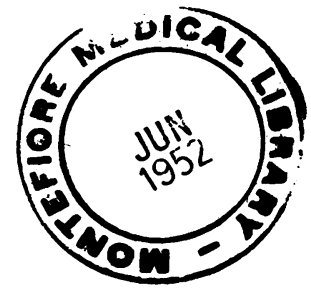

